\title{
SMA first approach Pancreaticoduodenectomy for cancer - should it be standard approach?
}

Binay Thakur ${ }^{1}$, Mukti Devkota ${ }^{1}$,Di Yonghui ${ }^{1}$, Yogesh Regmi ${ }^{2}$, Durga Khanal ${ }^{3}$, Kopila Khadaka ${ }^{3}$

${ }^{1}$ Department of Surgical Oncology, BP Koirala Memorial Cancer Hospital

${ }^{2}$ Department of Anesthesiology, BP Koirala Memorial Cancer Hospital

${ }^{3}$ Department of Nursing, BP Koirala Memorial Cancer Hospital

Correspondence: Dr. Binay Thakur- Chief, Department of Surgical Oncology, BP Koirala Memorial Cancer Hospital, Bharatpur, Nepal

Email- binaythakur@hotmail.com

\begin{abstract}
Background: pancreaticoduodenectomy (PD) has become the standard treatment for cancer of head of pancrease and periampullary malignancies. Recently superior mesenteric artery (SMA) first approach has shown to have several advantages over the conventional superior mesenteric vein (SMV) first approach. We present our initial experience with SMA first approach for PD.
\end{abstract}

Methods: Thirteen patients undergoing SMA first PD during year 2014-2015 (1 year) were prospectively studied. A posterior approach with "hanging maneuver" was used to identify SMA first and the resectibility was confirmed. Inferior pancreaticoduodenal artery (IPDA) was ligated before the division of pancreatic neck. Duct to mucosa pancreaticojejunostomy followed by hepaticojejunostomy and gastrojejunostomy was performed.

Results: patients with mean age of 53 years presented with abdominal pain and jaundice (100\%). Tumors were of periampullary region in 10 cases and of head of pancrease in three cases. Two patients required partial SMV wall resection with tangential repair. Mean operating time, blood loss and postoperative stay was 304 minutes, $380 \mathrm{ml}$ and 14 days, respectively. Replaced/ aberrant right hepatic artery from SMA was observed in $38 \%$ cases. Mean number of dissected nodes was 16 . R0 resection was achieved in $100 \%$ cases. There wasone postoperative mortality $(7.7 \%)$ and minor complications were noted in $46 \%$ cases.

Conclusion: SMA first PD helps to properly identify and control anomalous or accessory right hepatic artery arising from SMA, minimizes intraoperative bleeding due to proper control of in IPDA, facilitates easier SMV/ PV resection and provides the best chance for achieving Ro resection.

Key Words: pancreaticoduodenectomy; superior mesenteric artery; hanging maneuver. 